Head injury

\section{The role of helmets in skiing and snowboarding}

\section{P McCrory}

\section{Helmets need to be developed using injury data and suitable standards}

$\mathrm{H}$ aid was the first to raise the issue of helmet use in skiing when, in 1955, he published a study of fatal ski injuries treated in Innsbruck in Austria and suggested that head injury might be less severe if a helmet was worn. ${ }^{1}$ Since then the main proponent of helmet use in all aspects of skiing has been the Swiss neurosurgeon, Sooyoung Oh, although the evidence presented for his recommendations is anecdotal. ${ }^{23}$

The issue of sport specific helmet design becomes more important. Because at the present point in time we have only limited data on precise injury mechanisms in skiing and snowboarding, which suggests that there is no common mechanism to the occurrence of concussive injuries or skull fractures in these sports. ${ }^{45}$ This would mean that any helmet would have to be designed differently for these two sports. Injuries occurring in ski racing occur at far higher impact velocities than recreational skiing and furthermore usually occur on ice rather than snow. Once again, any potential helmet would have to be designed with these factors in mind. The ability of a conventional ski helmet to protect the brain from the velocities achieved in elite World Cup downhill racing is unachievable at present. Attention to other safety factors such as slope maintenance, flexible race gates, and crash barriers at races therefore becomes a more effective safety factor than helmet use.

Without valid helmet material and manufacturing standards, there is no guarantee that an off the shelf helmet has any protective capacity whatsoever. Furthermore depending on the nature and quality of the materials used, there is a potential for increasing cerebral injury rate significantly. The only international standard applicable for skiing helmets is the British standard BS EN 1077-1996, which sets out minimum standards requirements and helmets testing protocols for alpine skiers (including children) in competitions.

Issues about helmet maintenance and fit become important as well. This is especially true in children who may potentially be a group with the most to gain by wearing a suitable protective helmet. If the helmet does not fit firmly, then the protective capability is reduced. If the helmet is oversized and loose, as many parents may purchase a helmet with "room to grow" for their child, the chances of this being effective are small and potential problems in reducing vision and hearing may increase collision rates which is already the major injury mechanism in the paediatric age group.

Because of cost, helmets are usually made in two or three shell sizes with variations in liner thickness to accommodate a range of head sizes in each shell size. This means that the largest size in each shell is critical since its liner is the thinnest. The requirements of a size 8 helmet which must decelerate a $6.0 \mathrm{~kg}$ head are different to a size $63 / 4$ helmet which must protect a $4.2 \mathrm{~kg}$ head. The outer shell size remains the same in both cases but the liner thickness is the critical factor. Simple changes such as shifting head sizes into a larger shell may therefore protect greater numbers of participants. The problem is all too obvious in skiing where participants vary from small children through to adults and any proposed helmet must cope with this basic fact.

The possibility of a large helmet having a guillotining effect on the cervical spine needs to be explored. Until appropriate design standards exist, this risk must be viewed with some caution. There is evidence from the literature that children have a higher rate of neck and back injuries and the effect of helmets in contributing to this problem has not been adequately excluded. ${ }^{45}$
If a suitable helmet was available, then who would benefit? The highest risk groups would be ski racers, ski jumpers, freestyle aerialists, and children in all categories of skiing. The evidence that adult recreational skiers would benefit by helmets is not proven. Fortunately catastrophic ski injury, although tragic, is rare..$^{5-7}$ The risk of injury is reducing at all ski resorts over time and this may have more to do with slope maintenance and grooming, improved ski equipment, improved skill development through ski school instruction, skier fitness, and education. There is probably still much to do in terms of slope control-for example, restricting or removing ski passes from skiers who violate the skiers responsibility code by skiing too fast, out of control, or dangerously placing themselves as well as other slope users at risk.

At the present time no suitable helmet exists which is likely to protect all skiers and snowboarders. Although theoretically attractive in reducing head injury rates, the idea of skiers obtaining inferior and potentially dangerous helmets should not be encouraged. Better injury data analysis and suitable helmet standards are required to initiate the process of developing an appropriate protective helmet. Significant head injuries are rare in skiing and snowboarding and even if all participants wore helmets, it would not eliminate fatal injuries and may potentially increase the rate of other neurological injuries.

Br J Sports Med 2002;36:314

\section{Author's affiliations}

P McCrory, Centre for Sports Medicine Research and Education and the Brain research Institute, University of Melbourne, Melbourne, Australia

Correspondence to: $\mathrm{Dr}$ McCrory, $\mathrm{PO}$ Box 93 Shoreham, Victoria 3916, Australia; pmccrory@compuserve.com

\section{REFERENCES}

1 Haid B. Todliche skiverletzungen in einzugsgebeit der chirurgische universitatsklinik Innsbruck von 1944-1954. Arch Orthop Unfallchir 1955;47:105-14.

2 Oh S. Prevention of head injuries in skiing: mechanisms, experimental study and prevention. Basel: Karger, 1985.

3 Oh S, Reudi M. Depressed skull factures in skiing and their experimental study. Int J Sports Med 1982;3:168-73.

4 Sherry E, Korbel P, Henderson A. Children's skiing injuries in Australia. Med J Aust 1987; 147: 193-5.

5 Sherry E, Fenelon L. Trends in skiing injury type and rates in Australia: a review of 22,261 injuries over 27 years in the Snowy Mountains. Med J Aust 1991;155:513-15.

6 Prall J, Winston K, Brenan R. Severe snowboarding injuries. Injury 1995;26:539-42.

7 Johnson R. Skiing and snowboarding injuries. Postgrad Med 1990;88:36-51. 
Tendons

\section{Tendon healing: can it be optimised?}

N Maffulli, H D Moller, C H Evans

\section{Growth factors may be useful in tendon healing, possibly introduced using gene therapy}

T endon disorders are a major problem in sports and occupational medicine. Tendons have the highest tensile strength of all connective tissue because of a high proportion of collagen in the fibres and their closely packed parallel arrangement in the direction of force. The individual collagen fibrils are arranged into fascicles which contain blood vessels and nerve fibres. Specialised fibroblasts, tenocytes, lie within these fascicles and exhibit high structural organisation. ${ }^{1}$ Histologically, they appear as star shaped cells in cross sections. In longitudinal sections, they are arranged in rows following the direction of the tendon fibres. This specialised arrangement is related to their function, as tenocytes synthesise both fibrillar and nonfibrillar components of the extracellular matrix, and are able to reabsorb collagen fibrils. ${ }^{2}$ The fascicles themselves are enclosed by epitenon. This is surrounded by the paratenon, and the potential space between them is filled by a thin, lubricating film of fluid which allows gliding of the tendon during motion.

\section{BIOLOGY OF TENDON HEALING}

Tendon healing is classically considered to occur through extrinsic and intrinsic healing. The intrinsic model produces obliteration of the tendon and its tendon sheath. Healing of the defect involves an exudative and a formative phase which, on the whole, are very similar to those associated with wound healing. Extrinsic healing occurs through the chemotaxis of the specialised fibroblasts into the defect from the ends of the tendon sheath. ${ }^{4}$ The process can be divided into three phases: inflammation, repair, and organisation or remodelling. In the inflammatory phase, occurring three to seven days after the injury, cells migrate from the extrinsic peritendinous tissue such as the tendon sheath, periosteum, subcutaneous tissue, and fascicles, as well as from the epitenon and endotenon. ${ }^{5}$ Initially, the extrinsic response far outweighs the intrinsic one. This results in the rapid filling of the defect with granulation tissue, tissue debris, and haematoma. The migrating fibroblasts still play a phagocytic role, and are arranged in a radial fashion in relation to the direction of the fibres of the tendon. Biomechanical stability is given by fibrin.

The migrated fibroblasts begin to synthesise collagen around day 5. Initially, these collagen fibres are randomly orientated. Tenocytes become the main cell type, and over the next five weeks collagen is continuously synthesised During the 4th week, a noticeable increase in proliferation of fibroblasts of intrinsic origin, mainly from the endotenon, takes place. These cells take over the main role in the healing process and both synthesise and reabsorb collagen. The newly formed tissue starts to mature, and the collagen fibres are increasingly orientated along the direction of force through the tendon. This phase of repair continues for two months after the initial injury. Final stability is acquired during the remodelling induced by the normal physiological use of the tendon. This further orientates the fibres into the direction of force. In addition, cross linking between the collagen fibrils increases the tendon tensile strength. During the repair phase, the mechanically stronger type 1 collagen is produced in preference to type 3 collagen, thus slightly altering the initial ratio of these fibres to increase the strength of the repair. ${ }^{6}$

Despite intensive remodelling over the following months, complete regeneration of the tendon is never achieved. The tissue replacing the defect remains hypercellular. The diameter of the collagen fibrils is altered, favouring thinner fibrils with reduction in the biomechanical strength of the tendon.

In tendinopathic and ruptured Achilles tendons, there is a reduction in the proportion of type 1 collagen, and a significant increase in the amount of type 3 collagen, $^{7}$ responsible for the reduced tensile strength of the new tissue which is due to a reduced number of cross links compared with type 1 collagen. ${ }^{8}$ Recurring microinjuries lead to the development of hypertrophied biologically inferior tissue replacing the intact tendon.

\section{CYTOKINETIC MODULATION OF TENDON HEALING}

Growth factors and other cytokines play a key role in the embryonic differentiation of tissue and in the healing of tissues.' Growth factors stimulate cell proliferation and chemotaxis, and aid angiogenesis, influencing cell differentiation. They regulate cellular synthetic and secretory activity of components of extracellular matrix. Finally, they influence the process of wound healing.
In the normal flexor tendon of the dog, the levels of basic fibroblast growth factor are higher than the levels of platelet derived growth factor (PDGF). In injured tendons, the converse is true. ${ }^{10}$ Under the influence of PDGF, chemotaxis and the rate of proliferation of fibroblasts and collagen synthesis are increased." Fibroblasts of the patellar tendon show increased proliferation in vitro after the administration of basic fibroblast growth factor. ${ }^{12}$ In addition, an angiogenic effect is evident. ${ }^{13}$ During the embryogenesis of tendon, bone morphogenic proteins (BMP), especially BMP 12 and 13, cause increased expression of elastin and collagen type 1. Also, animal studies have shown that BMP 12 exerts a positive effect on the healing processes of the patellar tendon. ${ }^{14}$

The growth factors of the transforming growth factor $\beta$ superfamily induce an increase in mRNA expression of type l collagen and fibronectin in cell culture experiments. $^{15}$

High expression of collagen type 1 seems to be essential to achieve faster healing of tendons. Consequently, there should be a shift from the initial production of collagen type 3 to type 1 early in the healing process. The afore mentioned growth factors could potentially be used to influence the processes of regeneration of tendons therapeutically. However, it is unlikely that a single growth factor will give a positive result. The interaction of many factors present in the right concentration at the right time will be necessary.

\section{GENE THERAPY TO PROVIDE GROWTH FACTORS}

Growth factors have a limited biological half life. Given the complexity of the healing process of tendons, a single application of growth factors is unlikely to be successful. As there is no bioavailability of oral proteins, repeated local injections would be necessary to maintain levels in the therapeutic range. This can be technically difficult in operatively treated tendons. The transfer of genes for the relevant growth factors seems an elegant alternative. ${ }^{16}$ After cellular uptake and expression of genes, a high level of the mediators can be locally produced and secreted

To achieve this goal, vectors are used enabling the uptake and expression of genes into target cells. Vectors can be broadly grouped into viral and non-viral. Viral vectors are viruses deprived of their ability to replicate, into which the required genetic material can be inserted. They are effective, as the introduction of their genetic material into host cells forms part of their normal life cycle. Non-viral vectors have specific characteristics that enable penetration of the nucleus-for example, liposomal transport. The genes are released in the vicinity of the target cells without systemic dilution. 
There are two main strategies for transfer using vectors. In in vivo transfer, the vectors are applied directly to the relevant tissue. In vitro transfer involves removal of cells from the body, the gene transfer in vitro, and subsequent culture of these cells before they are reintroduced into the target site. Direct transfer is less invasive and technically easier, and can be started during treatment of the acute phase of the injury. A disadvantage is the non-specific infection of cells during the injection process. In addition, owing to the amount of extracellular matrix present, a vector with high transgenic activity is necessary to be able to transfer the gene to enough cells.

Indirect transfer of genes is safer. The relevant cell type is isolated and genetically modified. Before reintroduction into the body, cells can be selected and tested for quality. Owing to the work involved in this technique, it would be more suitable for the treatment of degenerative processes rather than acute injuries. The first studies on the feasibility of this procedure have been conducted using marker genes. ${ }^{17}$ The main gene used, lacZ, codes for the bacterial $\beta$ galactosidase which is not present in eukaryotic cells.

The addition of a suitable substrate changes the staining properties of the cells that express the new gene, enabling the effectiveness of transmission and the duration of expression of the foreign gene to be ascertained. With the vectors currently available, the gene is expressed for six to eight weeks in tendon tissue. ${ }^{18}$ Using this strategy, the transfer and expression of the PDGF gene into the patellar tendon of rats lead to an increase in angiogenesis and collagen synthesis in the tendon over four weeks. Gene expression of this duration could influence the whole healing process of tendons and could be the start of an optimised healing process.
In summary, tendon healing, even when successful, does not result in normal tendon. Mostly, the result is functionally satisfactory despite morphological differences and biomechanical weakness compared with a normal tendon. The therapeutic use of growth factors by gene transfer seems promising in the quest to produce a new tendon that is biologically, biomechanically, biochemically, and physiologically "normal".

Br J Sports Med 2002;36:315-316

\section{Authors' affiliations}

N Maffulli, Department of Trauma and Orthopaedic Surgery, Keele University School of Medicine, Staffordshire, UK

H D Moller, Orthopaedische Klinik, Medizinische Hochschule im Annastift e.V. Hannover, Germany

C H Evans, Center for Molecular Orthopaedics, Harvard Medical School, Boston, MA, USA

Correspondence to: Professor Maffulli, Department of Trauma and Orthopaedic Surgery, Keele University School of Medicine, North Staffordshire Hospital, Thornburrow Drive, Hartshill, Stoke on Trent, Staffordshire ST4 7QB, UK; osa14@keele.ac.uk

\section{REFERENCES}

1 Maffulli N, Benazzo F. Basic sciences of tendons. Sports Medicine and Arthroscopy Review 2000;8:1-5.

2 Birk DE, Zycband El, Woodruff S, et al. Collagen fibrillogenesis in situ: fibril segments become long fibrils as the developing tendon matures. Developmental Dynamics 1997;208:291-8.

3 Gigante A, Specchia N, Rapali S, et al. Fibrillogenesis in tendon healing: an experimental study. Bollettino della Societa' Italiana di Biologia Sperimentale 1996;72:203-10.

4 Wang ED. Tendon repair. Journal of Hand Therapy 1998;11:105-10.

5 Reddy GK, Stehno-Bittel L, Enwemeka CS. Matrix remodeling in healing rabbit Achilles tendon. Wound Repair and Regeneration 1999;7:518-27.

6 A comparison of the size distribution of collagen fibrils in connective tissues as a function of age and a possible relation between fibril size distribution and mechanical properties. Proc R Soc Lond B Biol Sci

1978;203:305-21.

7 Maffulli N, Ewen SW, Waterston SW, et al. Tenocytes from ruptured and tendinopathic achilles tendons produce greater quantities of type III collagen than tenocytes from normal achilles tendons. An in vitro model of human tendon healing. Am J Sports Med

2000;28:499-505.

8 Jozsa L, Reffy A, Kannus P, et al. Pathological alterations in human tendons. Arch Orthop Trauma Surg 1990;110:15-21.

9 Grotendorst GR. Growth factors as regulators of wound repair. Int J Tissue React 1988;10:337-44.

10 Duffy FJ Jr, Seiler JG, Gelberman RH, et al. Growth factors and canine flexor tendon healing: initial studies in uninjured and repair models. J Hand Surg [Am] 1995;20:645-9.

11 Pierce GF, Tarpley JE, Tseng J, et al. Detection of platelet-derived growth factor (PDGF)-AA in actively healing human wounds treated with recombinant PDGF-BB and absence of PDGF in chronic nonhealing wounds. J Clin Invest 1995;96:1336-50.

12 Chan BP, Chan KM, Maffulli N, et al. Effect of basic fibroblast growth factor. An in vitro study of tendon healing. Clin Orthop 1997;342:239-47.

13 Gabra N, Khayat A, Calabresi P, et al. Detection of elevated basic fibroblast growth factor during early hours of in vitro angiogenesis using a fast ELISA immunoassay. Biochem Biophys Res Commun 1994:205: 1423-30.

14 Enzura Y, Rosen V, Nifuji A. Induction of hypertrophy in healing patellar tendon by implantation of human recombinant BMP 12. J Bone Miner Res 1996;11:401

15 Ignotz RA, Massague J. Transforming growth factor-beta stimulates the expression of fibronectin and collagen and their incorporation into the extracellular matrix. J Biol Chem 1986;261:4337-45.

16 Moller HD, Evans CD, Robins PD, et al. Gene therapy in orthopaedic sports medicine. In: Chan KM, Fu FH, Maffulli N, et al, eds. Controversies in orthopaedic sports medicine. Hog Kong: Williams and Wilkins, 1988:577-88.

17 Lou J, Manske PR, Aoki M, et al Adenovirus-mediated gene transfer into tendon and tendon sheath. J Orthop Res 1996;14:513-17.

18 Nakamura N, Shino K, Natsuume T, et al. Early biological effect of in vivo gene transfer of platelet-derived growth factor (PDGF)-B into healing patellar ligament. Gene Therapy 1998;5:1165-70. 


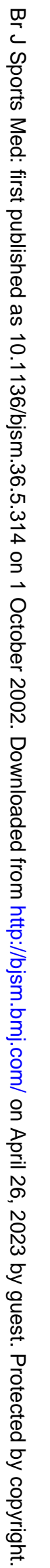




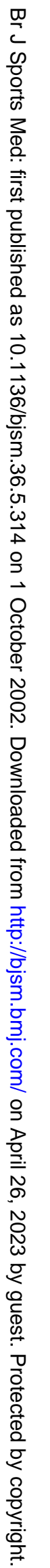




\section{Expression of concern about content of which Dr Paul McCrory is a single author}

This paper is authored by Dr Paul McCrory. During 2021 and 2022 there was an investigation by BJSM and BMJ which found that some of his work was the product of publication misconduct. Such misconduct includes plagiarism, duplicate publication, misquotation and misrepresentation in publications in respect of which he was listed as the sole author. ${ }^{1}$ We are placing a notice to readers on all content in relation to which he is identified as the sole author to alert them to the conclusions of our investigation.

(C) Author(s) (or their employer(s)) 2022. No commercial re-use. See rights and permissions. Published by BMJ.

Br J Sports Med 2022;0:1. doi:10.1136/bjsports-2022-106408eoc

D) Check for updates

\section{REFERENCE}

1 Macdonald H, Ragavooloo S, Abbasi K. Update into the investigation of former BJSM editor-in-chief Paul McCrory. Br I Sports Med 2022. 